\title{
Molecular dynamics at the receptor level of immunodominant myelin oligodendrocyte glycoprotein 35-55 epitope implicated in multiple sclerosis
}

\author{
Mary Patricia Yannakakis ${ }^{\mathrm{a}}$, Haralambos Tzoupis ${ }^{\mathrm{a}}$, Elena Michailidou ${ }^{\mathrm{a}}$, \\ Efthimia Mantzourani ${ }^{\mathrm{b}}$, Carmen Simal ${ }^{\mathrm{a}}$, Theodore Tselios ${ }^{\mathrm{a}, *}$ \\ a Department of Chemistry, University of Patras, GR-26504, Rion, Patras, Greece \\ ${ }^{\mathrm{b}}$ Cardiff University, Cardiff School of Pharmacy, CF10 3NB, Wales, UK
}

\section{A R T I C L E I N F O}

\section{Article history:}

Received 7 March 2016

Received in revised form 9 June 2016

Accepted 10 June 2016

Available online 18 June 2016

\section{Keywords:}

Bioactive conformation

Molecular dynamics

Multiple sclerosis (MS)

Myelin oligodendrocyte glycoprotein (MOG)

Human leukocyte antigen (HLA)

T-cell receptor (TCR)

\begin{abstract}
A B S T R A C T
Multiple Sclerosis (MS) is a common autoimmune disease whereby myelin is destroyed by the immune system. The disease is triggered by the stimulation of encephalitogenic T-cells via the formation of a trimolecular complex between the Human Leukocyte Antigen (HLA), an immunodominant epitope of myelin proteins and T-cell Receptor (TCR). Myelin Oligodendrocyte Glycoprotein (MOG) is located on the external surface of myelin and has been implicated in MS induction. The immunodominant 35-55 epitope of MOG is widely used for in vivo biological evaluation and immunological studies that are related with chronic Experimental Autoimmune Encephalomyelitis (EAE, animal model of MS), inflammatory diseases and MS. In this report, Molecular Dynamics (MD) simulations were used to explore the interactions of $\mathrm{MOG}_{35-55}$ at the receptor level. A detailed mapping of the developed interactions during the creation of the trimolecular complex is reported. This is the first attempt to gain an understanding of the molecular recognition of the $\mathrm{MOG}_{35-55}$ epitope by the HLA and TCR receptors. During the formation of the trimolecular complex, the residues $\mathrm{Arg}^{41}$ and $\mathrm{Arg}^{46}{ }^{4} \mathrm{MOG}_{35-55}$ have been confirmed to serve as TCR anchors while $\mathrm{Tyr}^{40}$ interacts with HLA. The present structural findings indicate that the Arg at positions 41 and 46 is a key residue for the stimulation of the encephalitogenic T-cells.
\end{abstract}

(C) 2016 Elsevier Inc. All rights reserved.

\section{Introduction}

Multiple Sclerosis (MS) is the most common autoimmune disease of the Central Nervous System (CNS) in which a coordinated attack of the immune system against myelin takes place $[1,2]$. Myelin Basic Protein (MBP), Proteolipid Protein (PLP), Myelin Oligodendrocyte Glycoprotein (MOG) and Myelin-Associated Glycoprotein (MAG) are the main myelin proteins in the CNS and they have been recognized as putative auto-antigens for MS [3]. MS onset is triggered by the activation of the encephalitogenic Tcells through the formation of a trimolecular complex between the T-cell receptor (TCR), the peptide (antigen) - with identical residue sequence to a fragment of a protein of the myelin sheath (molecular mimicry theory) [4,5] and the Major Histocompatibility Complex (MHC) or Human Leukocyte Antigen (HLA). The ability of the peptide-HLA complex to activate T-cells is correlated with

\footnotetext{
* Corresponding author.

E-mail address: ttselios@upatras.gr (T. Tselios).
}

the strength of its binding affinity with TCR [6-8]. Conversely, stimulation or not of T-cells that are responsible for MS follows [9-13]. The HLA class II receptors are dimers comprised of two different polypeptide chains ( $\alpha$ and $\beta$ ) [14,15]. In this class of macromolecules the polypeptide chains are joined together, creating a single receptor for the antigens to bind to. Subsequently, the newly formed complex is recognized by the receptors on the surface of T-cells. The formation of the trimolecular complex leads to the activation of the T-cells through a cascade of biochemical changes and the induction of the immune response to the antigen. The TCR is also composed of two different polypeptide chains. The two chains comprising the TCR ( $\alpha$ and $\beta$ ), include variable domains called Complementarity Determining Regions (CDRs). These domains are implicated in the recognition process between the TCR and the HLAantigen complex [16]. The diversity of the CDRs plays a crucial role in the recognition of the different antigens as they are presented by the HLA receptors. The CDRs comprising the TCR chains are very effective at screening the various antigens presented to the T-cells $[9,17]$. It has been estimated by in vivo experiments that the TCR unique structures in humans are over $>2.5 \times 10^{7}[17,18]$. Despite 
the diverse numbers and the rigorous selection process of T-cells in the thymus, $[19,20]$ there are cases where the selection process fails to single out T-cells reacting to self-antigens [21]. Thus, the failure in the thymic selection leads to self-reactive T-cells and induction of autoimmune disorders such as MS [22,23].

The myelin sheath consists of multiple proteins; MBP and PLP are two of the major component of the myelin sheath, while MOG and Myelin-associated Glycoprotein (MAG) are less abundant $[24,25]$. The total myelin proteins consist of only $0.05 \%-0.1 \%$ of MOG; [24,25] however MOG or MOG epitopes activate T-cell immune responses in Experimental Autoimmune Encephalomyelitis (EAE, animal model of MS) and have also been associated with MS induction [26,27]. There have been extensive studies of different fragments of MOG as autoimmune triggers [28-32]. The MOG epitopes 1-22, 61-80, 92-106 have been highlighted as target antigens and the 35-55 epitope has been found to induce highly specific antibodies reacting similar to the entire MOG protein $[33,34]$. Moreover, T-cell responses to MOG epitopes have been measured through biological assays in MS patients [29,30].

The $\mathrm{MOG}_{35-55}$ epitope ( $\mathrm{M}^{35}$ EVGWYRS $^{42}$ PFSRVVHLYRNGK ${ }^{55}$ ), based on the mouse/rat MOG peptide sequence, is shown as strongly immunogenic in mice leading to the development of chronic EAE [27,35]. Our group has previously rationally designed and synthesized linear and cyclic peptide analogues of human

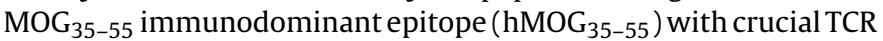
substitutions [26]. These altered peptides have proven to inhibit the clinical manifestation of symptoms of chronic EAE in mice [26]. The substitutions of Arg at positions 41 and 46 by Ala, result in peptide analogues that reduce the severity of MOG-induced EAE clinical symptoms in C57BL/6 mice when co-administered with mouse/rat MOG $_{35-55}$ peptide at the time of immunization [26]. The observed results justify the importance of Arg at positions 41, 46 for EAE induction [26].

To the best of our knowledge no conformational study of hMOG $_{35-55}$ epitope in complex with TCR and HLA has been reported. Hence, this is the first attempt to provide a deeper understanding of the structural requirements in the trimolecular complex with $\mathrm{hMOG}_{35-55}$ epitope. A detailed analysis of interactions between $\mathrm{hMOG}_{35-55}$ and the respective receptors (HLA and TCR) could provide valuable information for rational design of altered peptide ligands (APLs) and non-peptide mimetics with inhibitory activity. Herein, the structural properties of $\mathrm{hMOG}_{35-55}$ in three different environments were investigated, through the use of molecular dynamics simulations: i) we looked at the adopted conformations by the peptide in aqueous solution, ii) we carried out MD simulation studies using $\mathrm{hMOG}_{35-55}$ in combination with HLA DR2 (DRA, DRB1*1501) receptor and iii) the created trimolecular complex between $\mathrm{hMOG}_{35-55}$, HLA DR2 and TCR was explored. The investigation of the interactions between $\mathrm{hMOG}_{35-55}$ and the receptors in the trimolecular complex is expected to provide important information on the binding patterns of the hMOG epitope, which can assist researchers in the rational design of novel molecules, focusing on the inhibition of the stimulated encephalitogenic T-cells that are responsible for EAE and MS induction.

\section{Methods}

The high resolution crystal structure of HLA DR2 (DRA, DRB1*1501) in complex with the MBP ${ }_{83-96}$ antigen ( ${ }^{83}$ NPVVHFFKNIVTP ${ }^{96}$ ) and TCR was used for the MD simulations (PDB code: 1ymm) [36]. The $\mathrm{hMOG}_{35-55}$ epitope $\left(\mathrm{M}^{35}\right.$ EVGWYRP $^{42}$ PFSRVVHLYRNGK ${ }^{55}$ ), based on the human sequence, used for the simulation studies was constructed using PyMOL [37]. During this process the amino acids (L configuration), comprising the peptide, were placed in sequential order with no initial secondary structure assignment (unfolded conformation). The different systems were subjected to all-atom unrestrained MD simulations in explicit solvent using AMBER14 [38].

\subsection{Molecular dynamics (MD) simulation of $h M O G_{35-55}$}

For the construction of the $\mathrm{hMOG}_{35-55}$ peptide parameters, the AMBER force field ff14SB [39] has been used. The TIP3 P water model [40] was utilized for the solvation of the system and the total charge was neutralized by the addition of three $\mathrm{Cl}^{-}$ions. Truncated octahedral periodic boundary conditions have been applied to the system with a cutoff distance of $10 \AA$. The next step involved the minimization, followed by the heating of the system, under constant volume, to $300 \mathrm{~K}$ for $100 \mathrm{ps}$ using the Langevin dynamics temperature scaling [41]. This was followed by equilibration for another $100 \mathrm{ps}$ under constant pressure. Both heating and pressure equilibration were performed using a $10 \mathrm{kcal} \mathrm{mol}^{-1} \AA^{-2}$ restraint on the solute. The equilibration step under constant pressure was prolonged for a further $200 \mathrm{ps}$, after removing all restraints. The MD production run was performed under constant pressure and temperature conditions (NPT ensemble) for $100 \mathrm{~ns}$. The temperature was kept constant with the use of the Langevin thermostat (using a collision frequency of $2 \mathrm{ps}^{-1}$ ). All bonds involving hydrogen atoms were kept to their equilibrium distance with the SHAKE algorithm (allowing for a 2 fs time step to be used) [42]. The long range electrostatic interactions were calculated with the Particle Mesh Ewald (PME) method [43].

\subsection{Molecular dynamics (MD) simulation of the $h M O G_{35-55}$ complexed with the HLA DR2 receptor}

The positioning of the $\mathrm{hMOG}_{35-55}$ epitope inside the HLA DR2 receptor was performed manually using PyMOL [37]. The minimized structure (Section 2.1) of the linear hMOG $_{35-55}$ epitope was superimposed with the crystal structure of the MBP $83-96$ epitope (PDB code: 1ymm).[36] The orientation of hMOG $_{35-55}$ resembles the positioning of the $\mathrm{MBP}_{83-96}$ epitope inside the binding pocket of HLA DR2 $[36,44]$. The residues $\mathrm{Tyr}^{40}$, Pro $^{43}, \mathrm{Ser}^{45}$ and $\mathrm{Val}^{48}$ of hMOG were placed in the respective pockets (P1, P4, P6 and P9 for HLA DR2), as reported in the literature $[36,44,45]$. In the next step, the residues comprising the TCR were removed along with all crystallographic water molecules. The missing hydrogens were added using the tLeap module in AMBER14.

As with the MD simulation of hMOG $35-55$ in water, the parameters for the receptor were constructed using the AMBER force field ff14SB [39]. The total charge of the system was neutralized with the addition of fourteen $\mathrm{Na}^{+}$ions. The TIP3 P water model [40] was used in the solvation of the system and truncated octahedral periodic boundary conditions were applied to the system with a cutoff distances of $10 \AA$. The system was minimized over 5000 steps, followed by the gentle heating to 300 Kover 100 ps, using the Langevin dynamics temperature scaling (time step $2 \mathrm{fs}$ ). The system was further equilibrated under constant pressure for $100 \mathrm{ps}$. As mentioned in Section 2.1, both the heating and equilibration steps were performed using a $10 \mathrm{kcal} \mathrm{mol}^{-1} \AA^{-2}$ restraint on the solute. A $200 \mathrm{ps}$ equilibration step, under constant pressure, was performed following the removal of the restraint on the solute. Finally, an MD production run for $40 \mathrm{~ns}$ was performed using the NPT ensemble.

\subsection{Molecular dynamics (MD) simulation of TCR-hMOG $35-55-H L A$ DR2 complex}

For the MD simulation of the $\mathrm{hMOG}_{35-55}$ epitope in complex with both TCR and HLA the construction of the system followed the same steps described in Section 2.2. The minimized conformation of the linear hMOG $35-55$ epitope (Section 2.1) was superimposed with 
(a)

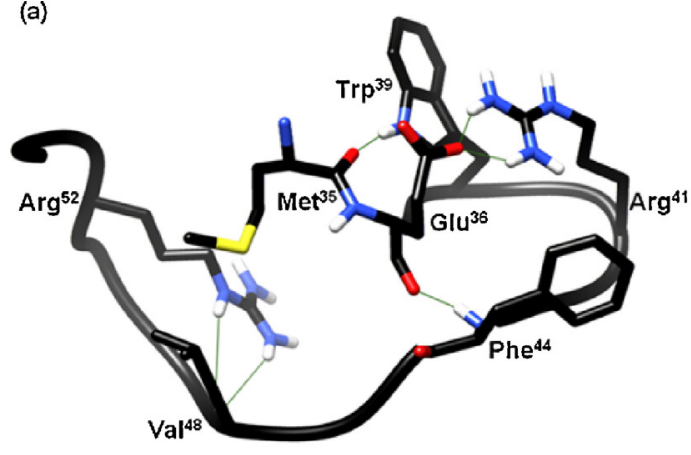

(b)

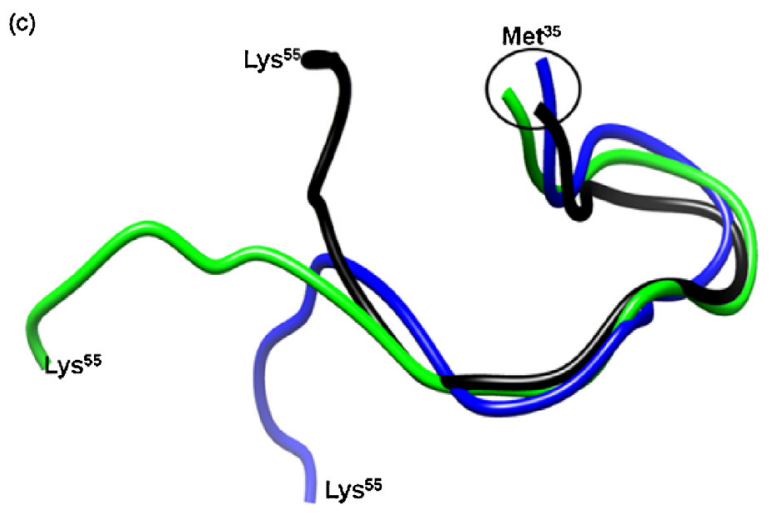

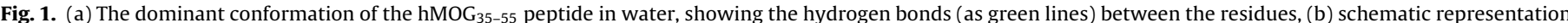

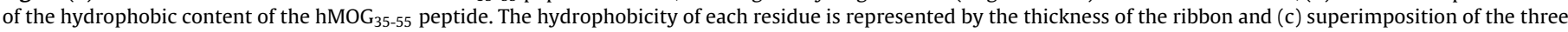

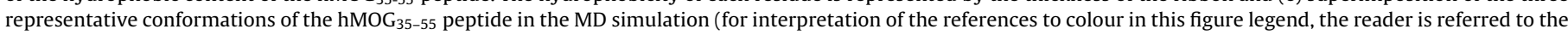
web version of this article).

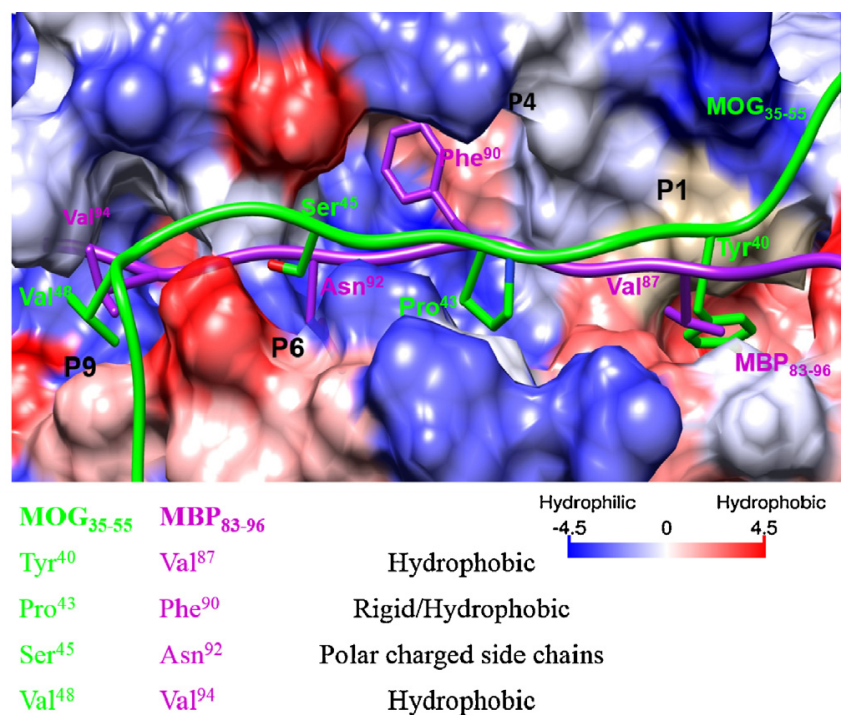

Fig. 2. The binding cavity, depicted as surface, of HLA DR2 shows the pockets P1, P4, $\mathrm{P} 6$ and P9 in the dominant representative conformation of the HLA DR2 - $\mathrm{hMOG}_{35-55}$ complex. The hMOG $_{35-55}$ (green) epitope is superimposed with the crystal structure of $\mathrm{MBP}_{83-96}$ (purple) taken from the crystal structure 1ymm. The peptides' backbone is represented as ribbon and the side chains of the residues are outlined as sticks. The surface of the receptor is coloured according to the Kyte-Doolittle hydrophobic index (for interpretation of the references to colour in this figure legend, the reader is referred to the web version of this article).

the crystal structure of HLA DR2/MBP $\left.{ }_{83-96} / \mathrm{TCR}\right)$. Thus, the TCR from the crystal structure was positioned over the hMOG $_{35-55}-\mathrm{HLA}$ DR2 complex. Subsequently, the $\mathrm{MBP}_{83-96}$ peptide was removed from the crystal structure along with the water molecules. The preparation of the system and the MD simulation followed the steps mentioned in Section 2.2. A total of thirteen $\mathrm{Na}^{+}$ions were used to neutralize the system and the solvation of the complex in explicit solvent was done using the TIP3 P water model [40].

A 40 ns MD production run in the created trimolecular complex was performed using AMBER14 [38]. Studies in similar biological systems show that the simulation time reported here is adequate for the conformational analysis of the bound epitope [46-50]. The parameters for the proteins and the peptide were constructed using the AMBER ff14SB force field [39]. The next step involved the minimization of the system followed by the heating of the complex to $300 \mathrm{~K}$ for $100 \mathrm{ps}$. Constant pressure equilibration was performed for a total of $300 \mathrm{ps}$. During the first $100 \mathrm{ps}$, a $10 \mathrm{kcal} \mathrm{mol}^{-1} \AA^{-2}$ restraint constant was imposed on the solute, which was then removed for the rest of the equilibration run. The MD production run was attained under isothermal and isobaric conditions and the temperature was kept constant with the use of the Langevin thermostat. The bonds involving hydrogen atoms were kept to their equilibrium distance with the SHAKE algorithm [42]. The Particle Mesh Ewald (PME) method ${ }^{43}$ was implemented for the calculation of the long range electrostatic interactions. Following, the above procedure, we also performed two MD simulations with mutated forms of hMOG $35-55$, namely: $\mathrm{hMOG}_{35-55}\left(\mathrm{Ala}^{41}\right)$ and $\mathrm{hMOG}_{35-55}\left(\mathrm{Ala}^{41,46}\right)$. In these variants, the Arg was replaced by Ala in position 41 and in positions $41 / 46$, respectively.

\subsection{Trajectory and clustering analysis}

The analysis (Rms deviations, atomic fluctuations and clustering calculations) of all the AMBER MD trajectories, was carried out using the cpptraj module [51] of the AMBER14 molecular package. The analysis of the hydrogen bond (HB) interactions was based on geometrical criteria, the limitations imposed were a $3.5 \AA$ cutoff for donor-acceptor distance and a donor-hydrogen-acceptor angle 
(a)
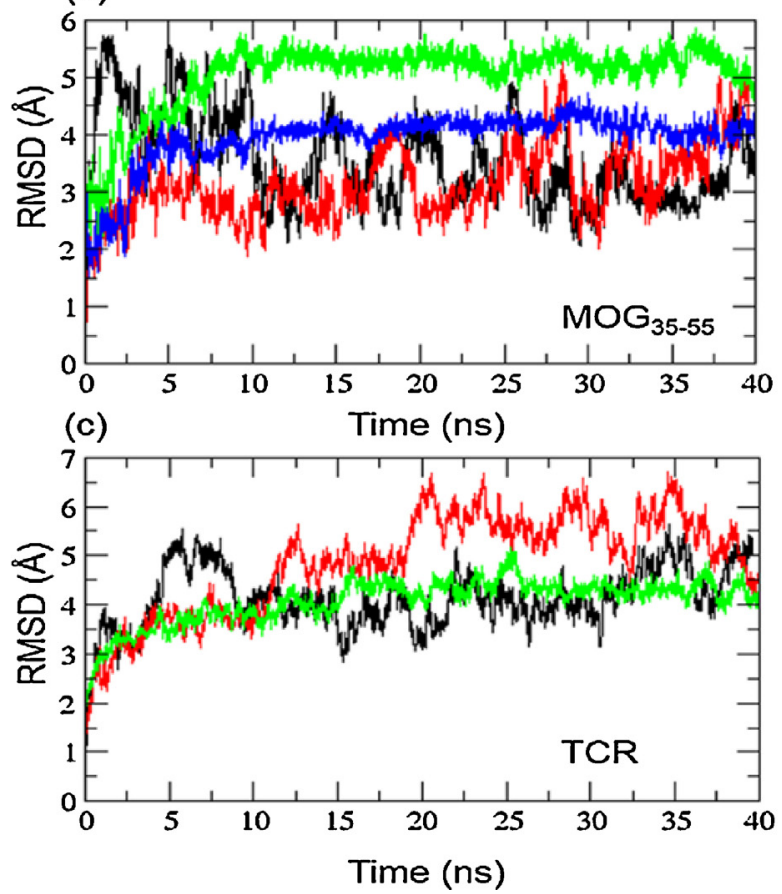

(b)
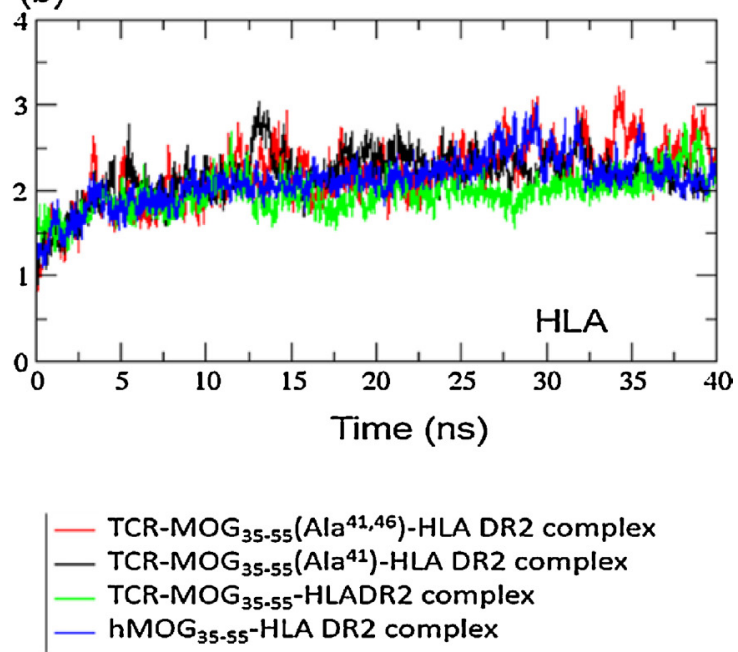

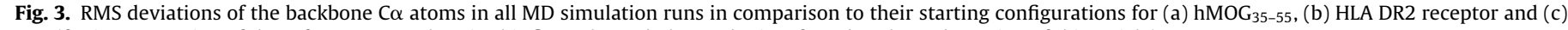
TCR (for interpretation of the references to colour in this figure legend, the reader is referred to the web version of this article).

cutoff of $120^{\circ}$. For the clustering analysis of the various MD trajectories, the hierarchical method [52] was used in the grouping process, with the Rms being the distance metric (cutoff $2.0 \AA$ ). The representative conformations presented in the paper are snapshots taken from the reference (centroid) simulation frames, used during the clustering analysis by the cpptraj module.

\section{Results}

\subsection{Conformational characteristics of $\mathrm{hMOG}_{35-55}$ epitope}

The analysis of the MD trajectory for the $\mathrm{hMOG}_{35-55}$ in solution revealed that $\mathrm{hMOG}_{35-55}$ does not retain an extended form. The different conformations adopted throughout the 100 ns trajectory, showed that the dominant form of the peptide presents two bends (Fig. 1a). The particular conformation is displayed on approximately $40 \%$ of the simulation time. The bend conformation of the peptide may be partly attributed to its hydrophobic content (Fig. 1b, thicker ribbon). The hydrophobic residues may force the polypeptide chain to seek conformations that decrease their exposure to the solvent. As expected, the small length does not allow the peptide to adopt a more stable secondary structure like $\alpha$-helix or $\beta$-sheet. Instead hMOG $_{35-55}$ remains in a largely unfolded state in aqueous solution, in accordance with experimental observations [53,54].

The adoption of a bend conformation by the peptide is further supported by the analysis of the secondary structures of the residues during the MD simulation (Fig. S1, in Supporting Information). It is evident that the secondary structure of the peptide does not present a stable conformational profile throughout the MD simulation (Fig. S1). Though, it displays certain helical features, such as bends and turns (Fig. S1, red and brown colours, respectively). These observations are in close agreement with those reported by Albouz-Abo et al. [53]. In the particular paper, Circular Dichroism (CD) data is reported in both the rat and human forms of $\mathrm{MOG}_{35-55}$. The CD experiments show evidence of helical conformations adopted mainly by the rat sequence and to a lesser extent by the human form of MOG in increased concentrations of trifluroethanol [53]. In our theoretical model (Fig. 1), the $\mathrm{hMOG}_{35-55}$ peptide presents a semi-closed conformation (random coil) as also reported by the study of Albouz-Abo et al. in aqueous solution [53]. The proposed conformation also presents, common features with the closed conformation reported for the rat/mouse $\mathrm{MOG}_{35-55}$ peptide by Ntountaniotis et al. [54] in both DMSO and aqueous solutions. The dominant form of $\mathrm{hMOG}_{35-55}$ (Fig. 1a, Fig. S2), displays two bends in the polypeptide backbone. The first bend is observed between residues $\operatorname{Trp}^{39}, \operatorname{Tyr}^{40}$ and $\operatorname{Arg}^{41}$ (Fig. $1 \mathrm{a}$ and Fig. S1 red colour) and the second in the area defined by residues Val $^{48}$ and $\mathrm{Arg}^{52}$ (Fig. S1, red colour). The particular conformation of the peptide is defined by the presence of extensive hydrogen bonds between the different amino acids. The most important interactions are those between residues $\mathrm{Glu}^{36}-\mathrm{Arg}^{41}$ and Glu $^{36}$-Phe ${ }^{44}$ (Fig. 1a, dark green lines). The hydrogen bond between the backbone carbonyl $-\mathrm{O}$ of $\mathrm{Glu}^{36}$ and the backbone $-\mathrm{NH}$ of Phe ${ }^{44}$ causes the peptide to form a loop, which is further stabilized by the interactions between the side chains of $\mathrm{Glu}^{36}$ and $\operatorname{Arg}^{41}$ (Fig. 1a). This loop appears almost throughout the simulation time as shown by the clustering analysis. Besides the above mentioned dominant form of hMOG $35-55$ (Fig. 1c, black), there are two more representative conformations (Fig. 1c, blue and green) in the MD simulation. Although both of these conformations retain the loop between residues 35-44, the second bend observed in the dominant form does not appear in these structures. The hydrogen bond between $\mathrm{Val}^{48}$ and $\mathrm{Arg}^{52}$ (Fig. 1a) is absent in these conformations leading to the loss of the second bend in the structure of the peptide.

\subsection{Conformational analysis of MMOG $_{35-55}-H L A$ DR2 receptor complex}

A visual inspection of all the conformations during the MD simulation was carried out and the amino acids of the $\mathrm{hMOG}_{35-55}$ with side chains that were oriented into the HLA DR2 main pockets were recorded (Fig. S3). The conformational analysis of the $\mathrm{hMOG}_{35-55^{-}}$ 
HLA DR2 complex showed that the peptide occupies pockets 1, 4, 6 and 9 of the receptor (Fig. S3). These four pockets are the same areas occupied by the $\mathrm{MBP}_{83-96}$ epitope (Fig. 2). The clustering anal-

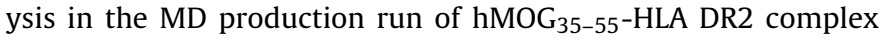
revealed that the dominant conformation of the peptide is the one depicted in Figs. 2 and S3. In fact, the particular conformation is found to be present for approximately $70 \%$ of the simulation time. This observation may suggest that the $\mathrm{hMOG}_{35-55}$ epitope favours a particular conformation when bound to HLA DR2 and retains this structure during most of the simulation time. The Rms deviations recorded during the MD simulation, show that both $\mathrm{hMOG}_{35-55}$ and the HLA receptor retains a stable configuration throughout the MD simulation (Fig. 3a and b, blue), with respect to their initial configuration.

A more detailed examination showed that in pockets 1,6 and 9, the residues interacting with the HLA DR2 receptor are similar in nature to those of the $\mathrm{MBP}_{83-96}$ epitope occupying the same space in the receptor (Fig. 3). For instance, $\mathrm{Tyr}^{40}\left(\mathrm{hMOG}_{35-55}\right)$ and $\mathrm{Val}^{87}$ $\left(\mathrm{MBP}_{83-96}\right)$ have both hydrophobic side chains and are oriented towards a buried area of the HLA receptor in pocket P1 (Fig. 2). The specific area of the receptor is rich in hydrophobic residues, such as $\mathrm{Phe}^{24}, \mathrm{Phe}^{26}, \mathrm{Ile}^{31}, \mathrm{Phe}^{54}$ and Val ${ }^{85}$ (Fig. 2, red surface colour). Moreover, the bulkier side chain of $\operatorname{Tyr}^{40}\left(\mathrm{hMOG}_{35-55}\right)$ is buried deeper in the particular pocket of HLA and may enhance the hydrophobic interactions between the epitope and the receptor, compared to $\mathrm{MBP}_{83-96}$. A similar trend is observed in the P6 pocket of HLA with the presence of $\operatorname{Ser}^{45}$ (hMOG $35-55$ ) and Asn $^{92}$ (MBP $83-96)$, which both present polar side chains. The -OH group from $\operatorname{Ser}^{45}$ (hMOG ${ }_{35-55}$ ) interacts with neighbouring residues such as $\mathrm{Glu}^{31}$, Asn ${ }^{62}$ and $\mathrm{Asp}^{66}$ of the HLA. Likewise, the amide side chain of $\mathrm{Asn}^{92}\left(\mathrm{MBP}_{83-96}\right)$ interacts with the same amino acids of the receptor in the P6 pocket. Finally, in $\mathrm{P9}$ pocket both $\mathrm{hMOG}_{35-55}$ and $\mathrm{MBP}_{83-96}$ have in the same position (48 and 94, respectively) a Val residue, surrounded by amino acids $\mathrm{Val}^{65}$ and $\mathrm{Ala}^{68}$ of the receptor (Fig. 2). The only difference between the two epitopes is focused in the P4 pocket of HLA DR2. In hMOG $35-55$ there is $\mathrm{Pro}^{43}$ in the P4 pocket of HLA while $\mathrm{MBP}_{83-96}$ has a hydrophobic residue $\left(\mathrm{Phe}^{90}\right)$ at the same position. The $\mathrm{P} 4$ pocket of the receptor includes residues with polar side chains, thus the smaller and restricted side chain of Pro may induce conformational changes in the residues surrounding $\mathrm{Pro}^{43}$ that may lead to a better positioning inside the $\mathrm{P} 4$ pocket of the HLA receptor [55].

\subsubsection{Hydrogen bond interactions in MMOG $_{35-55}-H L A$ DR2 receptor complex}

A detailed analysis of the hydrogen bonds (HBs), between the amino acids of $\mathrm{hMOG}_{35-55}$ and the HLA DR2 receptor, was carried out and the results are presented in Table 1. An important feature observed in the analysis of the MD simulation is the lack of water mediated hydrogen bonds between the peptide and the receptor. The binding cavity includes residues with bulky side chains such as $\mathrm{Asn}^{69}, \mathrm{Gln}^{71}, \mathrm{Arg}^{76}, \mathrm{His}^{81}$ and $\mathrm{Asn}^{82}$ in HLA (Table 1), that may prevent water molecules to move between receptor and epitope residues and consequently interact with either.

As observed in Table 1, hMOG 35 -55 creates an extensive hydrogen bond network with the HLA receptor. In pocket P1 of the HLA DR2, $\mathrm{Tyr}^{40}$ interacts with $\mathrm{His}^{81}$ from HLA anchoring the peptide in such a way to facilitate the interactions of its neighbouring residues (namely $\operatorname{Trp}^{39}, \mathrm{Arg}^{41}$ and Pro ${ }^{43}$ ). In fact Trp ${ }^{39}$ is involved in interactions with Arg ${ }^{80}$ in P1 pocket as well as Ser ${ }^{53}$ which is located in proximity to $\mathrm{P} 1$ pocket. Moreover, $\mathrm{Arg}^{41}$ due to its polar side chain, creates hydrogen bonds with three different amino acids of HLA (Gln ${ }^{70}$, Asp $^{76}$ and $\mathrm{Thr}^{77}$, Table 1 ) that are located in the P4 pocket and its vicinity, although such interactions are not appearing throughout the simulation time. This may be explained by the fact that the side chain of $\mathrm{Arg}^{41}$ is positioned towards the external area of the HLA receptor in a solvent exposed environment and may lead to extensive interactions with the TCR. Finally, Pro ${ }^{43}$ is interacting with $A s n^{62}$, located in the area between pockets P4 and P6 of the HLA, with the particular interaction being present throughout most of the simulation time. The particular HB interaction may enhance the HBs created between $\operatorname{Arg}^{41}$ and the receptor. Another residue of $\mathrm{hMOG}_{35-55}$, implicated in $\mathrm{HB}$ formation is $\mathrm{Ser}^{45}$, which forms extensive HBs with Asn ${ }^{62}$ and $A^{4} \mathrm{p}^{66}$ in $\mathrm{P} 6$ of the HLA receptor. Even residues such as $\mathrm{Arg}^{52}$ and $\mathrm{Lys}^{55}$ of the peptide, despite being positioned outside the binding areas P1, P4, P6 and P9 are interacting with residues of the HLA (Lys ${ }^{67}$ and $\mathrm{Gln}^{18}, \mathrm{Gly}^{20}$, respectively). These extensive interactions with the receptor may increase the binding affinity of the $\mathrm{hMOG}_{35-55}$.

As mentioned in Section 3.1, MMOG $_{35-55}$ adopts a bend conformation due to the presence of $\mathrm{HB}$ formed between residues such as $\mathrm{Glu}^{36}$ and Phe ${ }^{44}$ (Fig. 1). When bound to the HLA DR2 receptor, this conformational feature is not retained (Fig. 2) which is expected, since the interactions between the epitope and the residues of the receptor may lead to a conformational rearrangement of the epitope in order to enhance its binding. The bend aspect of the hMOG $_{35-55}$ peptide is an essential feature, because it allows the epitope to reach inside the receptor. Thus, residues such as $\mathrm{Tyr}^{40}$ and Pro $^{43}$ may interact with residues in different pockets (P1 and $\mathrm{P} 4$, respectively) and create a more extensive HB network with the HLA DR2 molecule.

\subsection{Conformational analysis of the trimolecular complex (TCR-hMOG $35-55-H L A$ DR2)}

As discussed in the Methods section (Section 2.3), the positioning of the $\mathrm{hMOG}_{35-55}$ epitope in the trimolecular complex was carried out via the superimposition of the epitope with the crystal structure of the $\mathrm{MBP}_{83-96}$ peptide. Thus, the amino acids of $\mathrm{hMOG}_{35-55}$ and $\mathrm{MBP}_{83-96}$ occupy similar pockets inside the TCR and HLA DR2 receptors. Furthermore, as reported in Section 3.2, hMOG $_{35-55}$ retains its initial positioning in the HLA DR2 pockets 1 , 4, 6 and 9 throughout the simulation. In that respect, residues such as $\mathrm{Arg}^{41}$ and mainly $\operatorname{Arg}^{46}$ of the peptide are facing towards the solvent when bound to HLA allowing the peptide to create enhanced interactions with the TCR during the antigen recognition process. This can be partially explained by the relative stable conformations observed in the trimolecular complex in the MD simulation. The Rms values for $\mathrm{hMOG}_{35-55}$ and the two receptors (HLA and TCR) after the first $5 \mathrm{~ns}$ of the simulation, remain relatively stable throughout the MD run (Fig. 3, green). This observation is in accordance with the conformational changes in the $\mathrm{hMOG}_{35-55}$-HLA DR2 complex. The conformational changes of the HLA receptor are similar in the trimolecular and hMOG $35-55$-HLA DR2 complexes (Fig. 3b, green and blue), as well as in the hMOG $_{35-55}$ epitope (Fig. 3a, green and blue). The difference, compared to the starting configuration, for the peptide in the two systems can be attributed to the interactions arising between $\mathrm{hMOG}_{35-55}$ and both receptors in the case of the trimolecular complex (Fig. 3a).

\subsubsection{Clustering analysis of the trimolecular complex (TCR-hMOG $35-55-H L A$ DR2)}

The clustering analysis showed that there are four representative clusters throughout the simulation (Fig. 4a). The different conformations of hMOG $35-55$ in their majority are exemplified by the bend conformation (Fig. $4 \mathrm{a}$, red). The particular conformation is appearing at $67 \%$ of the frames in our simulation. The other three clusters are present for a small percent of the simulation each, and are mostly appearing during the first $5 \mathrm{~ns}$, suggesting that the peptide prefers the bend conformation that is appearing on Pro ${ }^{42,43}$, in the presence of TCR and HLA. The aforementioned conformation may play a role in the orientation of $\mathrm{hMOG}_{35-55}$ in the binding 
Table 1

Residues in HLA DR2 receptor that are involved in HBs with those of the $\mathrm{hMOG}_{35-55}$ epitope, as recorded in all the MD simulations.

\begin{tabular}{|c|c|c|c|c|}
\hline \multirow[t]{3}{*}{ Peptide sequence $^{\mathrm{a}}$} & \multicolumn{4}{|l|}{ MD simulations $^{\mathrm{b}}$} \\
\hline & \multirow[t]{2}{*}{$\mathrm{hMOG}_{35-55}-\mathrm{HLA}^{\mathrm{c}}$} & \multicolumn{3}{|c|}{ TCR-hMOG $35-55$-HLA complexes } \\
\hline & & $\mathrm{hMOG}_{35-55}$ & $\mathrm{hMOG}_{35-55}\left(\mathrm{Ala}^{41}\right)$ & $\mathrm{hMOG}_{35-55}\left(\mathrm{Ala}^{41,46}\right)$ \\
\hline $\mathrm{Met}^{35}$ & - & - & - & Glu $^{87}$ \\
\hline $\mathrm{Glu}^{36}$ & - & - & - & $\operatorname{Arg}^{80}, \mathrm{Glu}^{87}$ \\
\hline $\mathrm{Val}^{37}$ & $\operatorname{Arg}^{80}$ & - & - & - \\
\hline $\operatorname{Trp}^{39}$ & $\operatorname{Arg}^{80}$ & $\operatorname{Thr}^{77}$, Arg $^{80}$ & $\operatorname{Arg}^{80}$ & - \\
\hline $\mathrm{Tyr}^{40}$ & $\mathrm{His}^{81}$ & $\mathrm{His}^{81}$, Asn $^{82}$ & - & Glu $^{55}$ \\
\hline $\operatorname{Arg}^{41}$ & $\mathrm{G} \ln 70$ & Glu $^{55}$ & $\mathrm{His}^{81}$ & $\operatorname{Tyr}^{78}$ \\
\hline Pro $^{43}$ & $\operatorname{Asn}^{62}$ & - & - & - \\
\hline $\mathrm{Phe}^{44}$ & - & $\mathrm{G} \ln ^{70}$ & $\mathrm{Gln} \ln ^{70}$ & $\mathrm{G} \ln ^{70}$ \\
\hline $\operatorname{Ser}^{45}$ & $\mathrm{Asp}^{66}, \mathrm{Asn}^{62}$ & - & $\operatorname{Asn}^{62}$ & $\operatorname{Asn}^{62}, \operatorname{Arg}^{13}$ \\
\hline $\operatorname{Arg}^{46}$ & - & $\operatorname{Asp}^{66}$ & $\mathrm{Gln}^{64}$ & - \\
\hline $\mathrm{Val}^{47}$ & - & - & - & $A s n^{69}$ \\
\hline $\mathrm{Val}^{48}$ & - & - & $\mathrm{Gln}^{64}$ & - \\
\hline $\mathrm{His}^{49}$ & - & Ala $^{61}$ & $\mathrm{Asp}^{66}$ & $\operatorname{Asn}^{69}$ \\
\hline Leu $^{50}$ & - & - & - & $\operatorname{Tyr}^{60}$ \\
\hline $\mathrm{Tyr}^{51}$ & Ala $^{64}$ & - & $\operatorname{Pro}^{56}$ & - \\
\hline $\operatorname{Arg}^{52}$ & Lys $^{67}$ & - & - & $\operatorname{Asp}^{57}$ \\
\hline Lys $^{55}$ & $\mathrm{G} \ln ^{18}$ & - & - & - \\
\hline
\end{tabular}

a Only the residues involved in hydrogen bonds are presented here.

b For detailed information on hydrogen bonds see Table S1 in Supporting Information.

c Only HBs with occurrence of more than $20 \%$ of the simulation time are presented here.

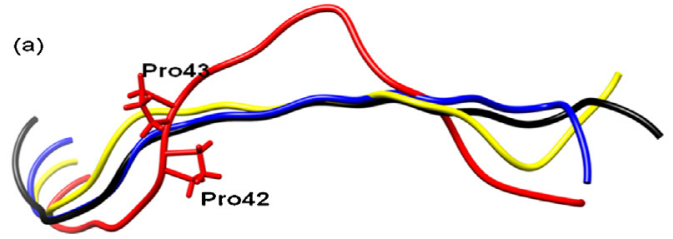

(b)

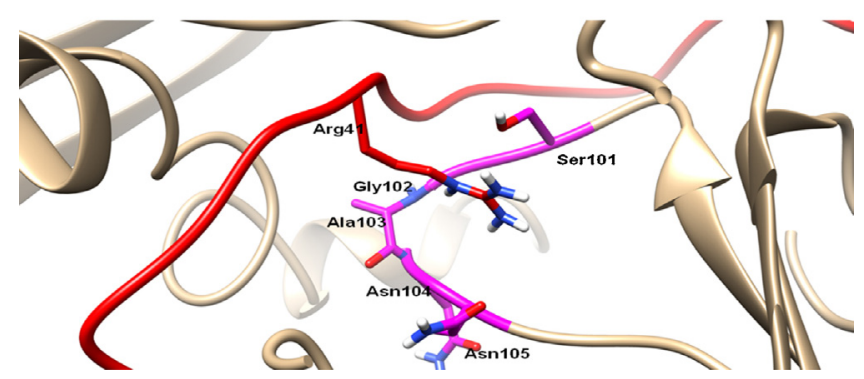

Fig. 4. (a) Representative structures of the 4 clusters generated from the MD simulation of the trimolecular complex (TCR-hMOG $35-55$-HLA DR2). In red is the representative conformation of the most dominant cluster (67\%) during the simulation. (b) The position of $\mathrm{Arg}^{41}$ (dominant cluster) in the TCR pockets P2, P3 and the proximity to residues 101-105 allow the Arg to form extensive HBs, as reported in Table 2 (for interpretation of the references to colour in this figure legend, the reader is referred to the web version of this article).

pocket of TCR. One such example is the positioning of $\mathrm{Arg}^{41}$ inside pockets P2 and P3 of TCR (Fig. 4b) that may lead to enhanced interactions with the TCR. Moreover, it may orient other residues of hMOG $_{35-55}$ in such a way so as to be correctly placed in the binding cavity of TCR. The orientation of the peptide in the TCR does not affect only the interactions between $\mathrm{hMOG}_{35-55}$ and TCR, but also the interactions with the HLA receptor.

\subsubsection{Hydrogen bond interactions in the trimolecular complex (TCR-hMOG $35-55-H L A$ DR2)}

It was observed in the hMOG $35-55$-HLA DR2 complex that $\mathrm{Tyr}^{40}$ (hMOG $_{35-55}$ ) is positioned similarly to $\mathrm{Val}^{89}\left(\mathrm{MBP}_{83-96}\right)$ and also creates HBs with the same amino acid - namely His ${ }^{81}$ - of HLA in the trimolecular complex (Table 1). Similarly, in the trimolecular complex, $\operatorname{Trp}^{39}$ (hMOG $_{35-55}$ ) participates in HB formation with residues $\left(\mathrm{Arg}^{80}, \mathrm{Thr}^{77}\right)$ inside pocket P1 of the HLA DR2 receptor (Table 1 ).
The interactions of $\mathrm{hMOG}_{35-55}$ with the TCR are reported in Table 2. In general, $\mathrm{hMOG}_{35-55}$ retains similar $\mathrm{HB}$ interactions when bound either to HLA or TCR and HLA together (Table 1) and the most important residues of the epitope are the two Arg in positions 41 and 46.

It has been theorised that Pro $^{42}$ in hMOG $_{35-55}$ plays a crucial role in the binding of the peptide to the TCR. The rigid region around Pro $^{42}-$ Pro $^{43}$ (Fig. 4a) seems to create a bend in the peptide. This structural feature allows the peptide to bend in a way that assists the interactions between $\operatorname{Arg}^{41}$ of the peptide and the TCR. In fact, $\mathrm{Arg}^{41}$ seems to reach easier into the pocket P3 of the TCR, in the presence of this bend. This conformational change may be the reason for the enhanced $\mathrm{HB}$ interactions of $\mathrm{Arg}^{41}$ with residues Gly ${ }^{102}$ and $\mathrm{Asn}^{104}$ in TCR. Also, it may lead to the HB formation between Arg $^{46}$ and residues Asp ${ }^{98}$, Thr ${ }^{100}$ and Glu $^{106}$ of the TCR. These interactions are present in the majority of the frames of the MD trajectory. $\operatorname{Arg}^{46}$ is positioned in the middle of $\mathrm{hMOG}_{35-55}$; hence the HBs formed with the TCR may further anchor the peptide firmly on the space between the two receptors. In this context, residues such as $\mathrm{Asn}^{53}$ that does not interact with any of the residues in the HLA receptor forms hydrogen bonds with $\mathrm{Gln}^{29}$ and $\mathrm{Thr}^{75}$ of the TCR (Table 2). These interactions are also present for most of the simulation time.

\subsubsection{Conformation characteristics of mutated $h M O G_{35-55}\left(\right.$ Ala $\left.^{41}\right)$ and $h M O G_{35-55}\left(\right.$ Ala $\left.^{41,46}\right)$ analogues}

The conformational analysis of the $\mathrm{hMOG}_{35-55}\left(\mathrm{Ala}^{41}\right)$ and $\mathrm{hMOG}_{35-55}\left(\mathrm{Ala}^{41,46}\right)$ analogues, revealed that the conformational changes in the peptide are more evident in the two variations of hMOG $_{35-55}$ compared to the hMOG $35-55$ epitope (Fig. 3a, red and black). The mutation in positions 41 and 46 of the $\mathrm{hMOG}_{35-55}$, affects the conformations of the peptide in the trimolecular complex. The substitution of Arg with Ala in both cases leads to loss of interactions between the peptide and the two receptors (HLA, TCR). The mutations and the subsequent change in the interactions formed by the peptide may explain the greater fluctuations with respect to the initial conformation of the RMS values in Fig. 3a (red and blue) over time compared to the $\mathrm{hMOG}_{35-55}$ epitope (Fig. 3a, green). Moreover, the clustering analysis performed in the two variants of the hMOG $35-55$ epitope, showed that there is a domi- 
Table 2

TCR residues that interact with residues of the $\mathrm{hMOG}_{35-55}$ epitope for the formation of respective HBs, as recorded in all the MD simulations.

\begin{tabular}{|c|c|c|c|}
\hline \multirow{2}{*}{$\begin{array}{l}\text { Peptide } \\
\text { sequence }^{\mathrm{a}}\end{array}$} & \multicolumn{3}{|c|}{ 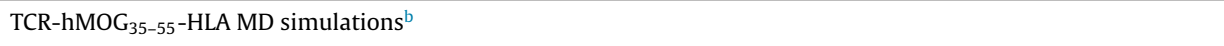 } \\
\hline & $\mathrm{hMOG}_{35-55}$ & $\mathrm{hMOG}_{35-55}\left(\mathrm{Ala}^{41}\right)$ & $\mathrm{hMOG}_{35-55}\left(\mathrm{Ala}^{41,46}\right)$ \\
\hline Met $^{35}$ & Thr $^{94}$ & - & - \\
\hline $\mathrm{Glu}^{36}$ & $\operatorname{Ser}^{28}$ & - & $\operatorname{Ser}^{28}$ \\
\hline Gly $^{38}$ & $\operatorname{Ser}^{95}$ & $\operatorname{Ser}^{95}$ & $\operatorname{Ser}^{95}$ \\
\hline $\operatorname{Trp}^{39}$ & - & - & Ala $^{103}$, Gly $^{96}$ \\
\hline $\mathrm{Tyr}^{40}$ & $\operatorname{Tyr}^{98}$ & Tyr $^{98}$ & - \\
\hline $\operatorname{Arg}^{41}$ & Gly $^{102}$, Asn $^{104}$ & - & - \\
\hline $\operatorname{Ser}^{45}$ & - & Thr $^{100}$ & - \\
\hline $\operatorname{Arg}^{46}$ & Asp $^{98}$, Thr $^{100}$, Glu $^{106}$ & Asp $^{98}$, Thr $^{100}$, Gly $^{106}$ & - \\
\hline $\operatorname{Arg}^{52}$ & $\operatorname{Ser}^{25}$ & - & - \\
\hline Asn $^{53}$ & $\operatorname{Gln}^{29}, \operatorname{Thr}^{75}$ & - & - \\
\hline Gly $^{54}$ & - & - & - \\
\hline Lys $^{55}$ & $\operatorname{Arg}^{24}$ & - & - \\
\hline
\end{tabular}

a Only the residues involved in hydrogen bonds are presented here.

b For detailed information on hydrogen bonds see Table S2 in Supporting Information.

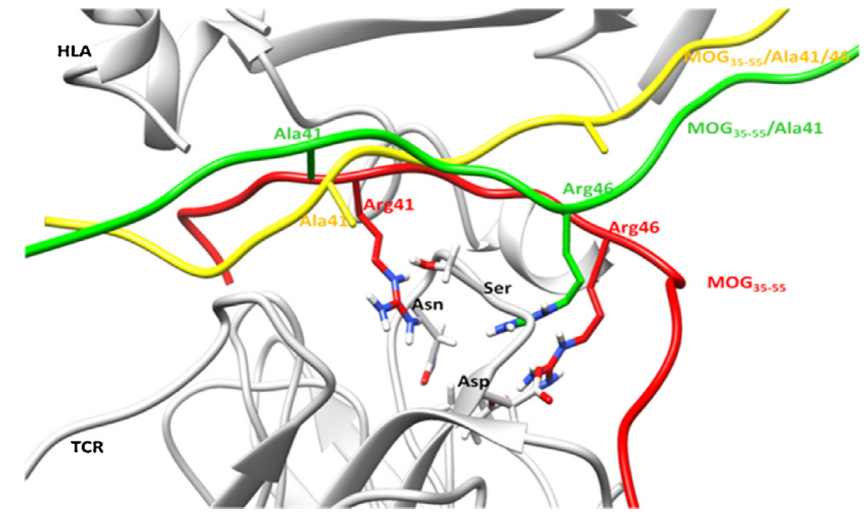

Fig. 5. The dominant representative conformations of the $\mathrm{hMOG}_{35-55}$ (red) and the two variants hMOG $35-55\left(\mathrm{Ala}^{41}\right)$ (green) and $\mathrm{hMOG}_{35-55}\left(\mathrm{Ala}^{41,46}\right)$ (yellow) at the interface between the HLA receptor (top) and TCR (bottom). The residues at positions 41 and 46 are presented as sticks while the rest of the proteins are presented as ribbon (for interpretation of the references to colour in this figure legend, the reader is referred to the web version of this article).

nant conformation for the peptides inside the trimolecular complex (approximately $72 \%$ of the simulation time) as presented in Fig. 5.

The analysis of the hydrogen bond interactions between the two mutated analogues of $\mathrm{hMOG}_{35-55}$, showed a loss of interactions with the receptors mainly with TCR (Table 2). As expected the particular loss is centered on the positions with the mutated amino acids (positions 41 and 46). The replacement of Arg by Ala causes the loss of the HBs observed in hMOG $35-55$ (Tables 2), that could be attributed to the increased polarity of Arg due to the guanidino group that can create an extensive interaction pattern. On the other hand, Ala bears a less bulky group, as a side chain, with no potential for creating hydrogen bonds. This mutation may cause indivertibly, conformational changes in the positioning of the peptide inside the cavity of the two receptors. As presented in Fig. 5 (red), the dominant conformation of the wt-hMOG $35-55$ peptide is more bent when compared to the representative conformations adopted during the MD simulations by $\mathrm{hMOG}_{35-55}\left(\mathrm{Ala}^{41}\right)$ (Fig. 5, green) and $\mathrm{hMOG}_{35-55}\left(\mathrm{Ala}^{41,46}\right)$ (Fig. 5, yellow). The Arg residues in positions 41 and 46 are found on either side of a loop in the TCR binding site with polar residues such as $A_{s p}{ }^{98}, \operatorname{Ser}^{101}$ and $A_{s n}{ }^{104}$. These polar residues interact with $\mathrm{Arg}$ in the $\mathrm{hMOG}_{35-55}$ epitope and assist to anchor the peptide in the TCR receptor. These interactions are not present on the $\mathrm{hMOG}_{35-55}\left(\mathrm{Ala}^{41}\right)$ and $\mathrm{hMOG}_{35-55}\left(\mathrm{Ala}^{41,46}\right)$ variants.

Additionally, a significant aspect observed in the three different MD simulations of the trimolecular complex (TCR-hMOG $35-55-\mathrm{HLA}$ DR2 receptor) is the retention of the interactions of Phe at position
44 of the hMOG $35-55$ epitope. In both variants of hMOG $35-55$ and in the $\mathrm{hMOG}_{35-55}$, the particular residue forms hydrogen bonds with $\mathrm{Gln}^{70}$ in the HLA receptor (Table 1) that are present throughout the MD simulation.

\section{Discussion}

The theoretical analysis based on the MD simulations, shows that $\mathrm{hMOG}_{35-55}$ can be an important activator of T-cells. The conformational analysis inside the HLA receptor and in the trimolecular complex revealed similar positioning with the $\mathrm{MBP}_{83-96}$ peptide, suggesting a comparable mode of interaction between the two epitopes and the two receptors. In regard to the interactions between the epitopes and the HLA-DR2 receptor, we observe that the residues involved in HBs have similar chemical properties (Fig. 2). These similarities (hydrophobic nature, charged side chains) may provide valuable information regarding the type of interactions between the different epitopes and the receptor. Thus, it is possible to cluster the interactions of the epitopes in groups and identify common patterns. The comparison of these interactions between the hMOG $_{35-55}$ epitope and the HLA are comparable to those of $\mathrm{MBP}_{83-96}$ (Fig. 2), nevertheless these two antigens exhibit a sequence similarity of only $14 \%$. This may be partially explained by the fact that the residues interacting with the receptor have similar side chains (Fig. 2).

The majority of HBs between the hMOG $35-55$ epitope and the HLA DR2 receptor stems mainly from residues $\operatorname{Trp}^{39}$ and $\mathrm{Arg}^{41}$. The same Arg also presents a high number of interactions with the TCR, suggesting that the particular residue plays a critical role in antigen identification by T-cells. It can also be deduced that the approach of TCR towards the peptide/HLA DR2 receptor complex leads to the increase of the HBs inside the newly formed trimolecular complex. Subsequently, this might indicate that the antigen binding affinity is amplified once it is placed between the two receptors.

We concluded that the key residues that enable the interactions between $\mathrm{hMOG}_{35-55}$ and the TCR are $\operatorname{Arg}^{41}$ and $\mathrm{Arg}^{46}$. The Arg residues at positions 41 and 46 of the peptide create an extensive HB network with both receptors. The substitution of Arg, at positions 41 and 46 of the epitope, with Ala leads to changes in the interactions of the peptide. Ala, in contrast to Arg, has a very small, non-polar side chain group that subsequently may affect the potential of the peptide to interact with TCR, since the particular position is solvent exposed and thus favours the presence of hydrophilic residues. As observed with the two variants of $\mathrm{hMOG}_{35-55}\left[\mathrm{hMOG}_{35-55}\left(\mathrm{Ala}^{41}\right)\right.$ and $\left.\mathrm{hMOG}_{35-55}\left(\mathrm{Ala}^{41,46}\right)\right]$, the mutation of Arg to Ala leads to loss of interactions between the TCR and hMOG $35-55$ (Table 2), while retaining the same pattern of 
HBs and binding affinity towards the HLA receptor (Table 1). In both the hMOG $_{35-55}$ and its mutations, the interactions between $\mathrm{Phe}^{44}$ and the HLA receptors are identical in nature.

\section{Conclusion}

The peptide variants $\left[\mathrm{hMOG}_{35-55}\left(\mathrm{Ala}^{41}\right)\right.$ and $\mathrm{hMOG}_{35-55}\left(\mathrm{Ala}^{41,46}\right)$ ] have proven to act as inhibitors of EAE, which is the animal model for MS [26]. The information compiled during our studies confirms that the mutation of key residues in $\mathrm{hMOG}_{35-55}$ leads to competitive binding to the HLA receptor and loss of key interactions with the TCR, thus preventing the formation of the trimolecular complex. This report provides a comprehensive theory regarding the interactions and conformational changes observed in the formation of trimolecular complex (TCR$\mathrm{hMOG}_{35-55}$-HLA DR2 receptor) and highlights key interactions of the hMOG $35-55$ epitope.

\section{Conflicts of interest}

The authors declare no conflict of interest.

\section{Acknowledgements}

This work is financially supported by the "Cooperation" Program 09SYN21-609 and by "Cooperation Greece-Israel Program" ISR-31480.P. Competitiveness \& Entrepreneurship (EPAN II), ROP Macedonia- Thrace, ROP Crete and Aegean Islands, ROP ThessalyMainland Greece- Epirus, ROP Attica. We also thank FICYT and the European Union (7th WP) for a Marie Curie CLARIN-cofund fellowship to CS.

\section{Appendix A. Supplementary data}

Supplementary data associated with this article can be found, in the online version, at http://dx.doi.org/10.1016/j.jmgm.2016.06. 005.

\section{References}

[1] L. Steinman, Multiple sclerosis: a coordinated immunological attack against myelin in the central nervous system, Cell 85 (1996) 299-302.

[2] M. Sospedra, R. Martin, Immunology of multiple sclerosis, Annu. Rev. Immunol. 23 (2005) 683-747.

[3] K.J. Smith, J. Pyrdol, L. Gauthier, D.C. Wiley, K.W. Wucherpfennig, Crystal structure of HLA-DR2 (DRA*0101 DRB1*1501) complexed with a peptide from human myelin basic protein, J. Exp. Med. 188 (1998) 1511-1520.

[4] N. Shahrizaila, N. Yuki, Guillain-barre syndrome animal model: the first proof of molecular mimicry in human autoimmune disorder, J. Biomed. Biotechnol. (2011) (2011) 829129.

[5] L. Moise, S. Beseme, R. Tassone, R. Liu, F. Kibria, F. Terry, et al., T cell epitope redundancy: cross-conservation of the TCR face between pathogens and self and its implications for vaccines and auto-immunity, Expert Rev. Vaccines 15 (2016) 607-617.

[6] A. Compston, A. Coles, Multiple sclerosis, Lancet 359 (2002) 1221-1231.

[7] M.M. Davis, J.J. Boniface, Z. Reich, D. Lyons, J. Hampl, B. Arden, et al., Ligand recognition by alpha beta T cell receptors, Annu. Rev. Immunol. 16 (1998) 523-544.

[8] B. Wootla, M. Eriguchi, M. Rodriguez, Is multiple sclerosis an autoimmune disease? Autoimmune Dis. (2012) (2012) 969657.

[9] B.J. Hare, D.F. Wyss, M.S. Osburne, P.S. Kern, E.L. Reinherz, G. Wagner, Structure, specificity and CDR mobility of a class II restricted single-chain T-cell receptor, Nat. Struct. Biol. 6 (1999) 574-581.

[10] M.G. Rudolph, I.A. Wilson, The specificity of TCR/pMHC interaction, Curr. Opin. Immunol. 14 (2002) 52-65.

[11] M.G. Rudolph, R.L. Stanfield, I.A. Wilson, How TCRs bind MHCs, peptides, and coreceptors, Annu. Rev. Immunol. 24 (2006) 419-466.

[12] H. Lassmann, Axonal and neuronal pathology in multiple sclerosis: what have we learnt from animal models, Exp. Neurol. 225 (2010) 2-8.

[13] E. Reuter, R. Gollan, N. Grohmann, M. Paterka, H. Salmon, J. Birkenstock, et al., Cross-recognition of a myelin peptide by CD8+ T cells in the CNS is not sufficient to promote neuronal damage, J. Neurosci. 35 (2015) 4837-4850.
[14] D.R. Madden, The three-dimensional structure of peptide-MHC complexes, Annu. Rev. Immunol. 13 (1995) 587-622.

[15] E.J. Adams, A.M. Luoma, The adaptable major histocompatibility complex (MHC) fold: structure and function of nonclassical and MHC class I-like molecules, Annu. Rev. Immunol. 31 (2013) 529-561.

[16] Y. Feng, J. van der Veeken, M. Shugay, E.V. Putintseva, H.U. Osmanbeyoglu, S. Dikiy, et al., A mechanism for expansion of regulatory T-cell repertoire and its role in self-tolerance, Nature 528 (2015) 132-136.

[17] X. Yang, M. Gao, G. Chen, B.G. Pierce, J. Lu, N.P. Weng, et al., Structural basis for clonal diversity of the public T cell response to a dominant human cytomegalovirus epitope, J. Biol. Chem. 290 (2015) 29106-29119.

[18] T.P. Arstila, A. Casrouge, V. Baron, J. Even, J. Kanellopoulos, P. Kourilsky, A direct estimate of the human alphabeta T cell receptor diversity, Science 286 (1999) 958-961.

[19] L. Hesnard, F. Legoux, L. Gautreau, M. Moyon, O. Baron, M.C. Devilder, et al., Role of the MHC restriction during maturation of antigen-specific human T cells in the thymus, Eur. J. Immunol. 46 (2016) 560-569.

[20] M.W. Buckley, P.C. Trampont, S. Arandjelovic, A.M. Fond, I.J. Juncadella, K.S. Ravichandran, ShcA regulates late stages of T cell development and peripheral CD4+ T cell numbers, J. Immunol. 194 (2015) 1665-1676.

[21] E. Gianchecchi, D.V. Delfino, A. Fierabracci, Recent insights on the putative role of autophagy in autoimmune diseases, Autoimmun. Rev. 13 (2014) 231-241.

[22] H.D. Chang, A. Radbruch, Targeting pathogenic T helper cell memory, Ann. Rheum. Dis. 70 (Suppl. (1)) (2011) i85-i87.

[23] C.J. Lessard, J.A. Ice, I. Adrianto, G.B. Wiley, J.A. Kelly, P.M. Gaffney, et al., The genomics of autoimmune disease in the era of genome-wide association studies and beyond, Autoimmun. Rev. 11 (2012) 267-275.

[24] O. Jahn, S. Tenzer, H.B. Werner, Myelin proteomics: molecular anatomy of an insulating sheath, Mol. Neurobiol. 40 (2009) 55-72.

[25] J. Patzig, O. Jahn, S. Tenzer, S.P. Wichert, P. de Monasterio-Schrader, S. Rosfa, et al., Quantitative and integrative proteome analysis of peripheral nerve myelin identifies novel myelin proteins and candidate neuropathy loci, J. Neurosci. 31 (2011) 16369-16386.

[26] T. Tselios, M. Aggelidakis, A. Tapeinou, V. Tseveleki, I. Kanistras, D. Gatos, et al., Rational design and synthesis of altered peptide ligands based on human myelin oligodendrocyte glycoprotein 35-55 epitope: inhibition of chronic experimental autoimmune encephalomyelitis in mice, Molecules 19 (2014) 17968-17984

[27] C. Delarasse, P. Smith, D. Baker, S. Amor, Novel pathogenic epitopes of myelin oligodendrocyte glycoprotein induce experimental autoimmune encephalomyelitis in C57BL/6 mice, Immunology 140 (2013) 456-464.

[28] N. Kerlero de Rosbo, R. Milo, M.B. Lees, D. Burger, C.C. Bernard, A. Ben-Nun, Reactivity to myelin antigens in multiple sclerosis: peripheral blood lymphocytes respond predominantly to myelin oligodendrocyte glycoprotein, J. Clin. Invest. 92 (1993) 2602-2608.

[29] M. Varrin-Doyer, A. Shetty, C.M. Spencer, U. Schulze-Topphoff, M.S. Weber, C.C. Bernard, et al., MOG transmembrane and cytoplasmic domains contain highly stimulatory T-cell epitopes in MS, Neurol. (R) Neuroimmunol. Neuroinflamm. 1 (2014) e20.

[30] A. Shetty, S.G. Gupta, M. Varrin-Doyer, M.S. Weber, T. Prod'homme, N. Molnarfi, et al., Immunodominant T-cell epitopes of MOG reside in its transmembrane and cytoplasmic domains in EAE, Neurol. (R) Neuroimmunol. Neuroinflamm. 1 (2014) e22.

[31] D. Linares, P. Mana, M. Goodyear, A.M. Chow, C. Clavarino, N.D. Huntington, et al., The magnitude and encephalogenic potential of autoimmune response to MOG is enhanced in MOG deficient mice, J. Autoimmun. 21 (2003) 339-351.

[32] G. Krishnamoorthy, A. Saxena, L.T. Mars, H.S. Domingues, R. Mentele, A Ben-Nun, et al., Myelin-specific T cells also recognize neuronal autoantigen in a transgenic mouse model of multiple sclerosis, Nat. Med. 15 (2009) 626-632

[33] G. Pacini, M. Ieronymaki, F. Nuti, G. Sabatino, M. Larregola, R. Aharoni, et al. Epitope mapping of anti-myelin oligodendrocyte glycoprotein (MOG) antibodies in a mouse model of multiple sclerosis: microwave-assisted synthesis of the peptide antigens and ELISA screening, J. Pept. Sci. 22 (2016) $52-58$.

[34] J.T. Mony, R. Khorooshi, T. Owens, MOG. extracellular domain (p1-125) triggers elevated frequency of CXCR3+ CD4+ Th1 cells in the CNS of mice and induces greater incidence of severe EAE, Mult. Scler. 20 (2014) 1312-1321.

[35] S. Bittner, A.M. Afzali, H. Wiendl, S.G. Meuth, Myelin oligodendrocyte glycoprotein (MOG35-55) induced experimental autoimmune encephalomyelitis (EAE) in C57BL/6 mice, J. Vis. Exp. (JoVE) 86 (2014) e51275, http://dx.doi.org/10.3791/51275.

[36] M. Hahn, M.J. Nicholson, J. Pyrdol, K.W. Wucherpfennig, Unconventional topology of self peptide-major histocompatibility complex binding by a human autoimmune T cell receptor, Nat. Immunol. 6 (2005) 490-496.

[37] J. Pokorna, L. Machala, P. Rezacova, J. Konvalinka, Current and novel inhibitors of HIV protease, Viruses 1 (2009) 1209-1239.

[38] D.A. Case, J.T. Berryman, R.M. Betz, D.S. Cerruti, T.E. Cheatham, T.A. Darden, et al., Amber 2015, University of California, San Francisco, CA, 2015.

[39] J.A. Maier, C. Martinez, K. Kasavajhala, L. Wickstrom, K.E. Hauser, C. Simmerling, ff14SB: improving the accuracy of protein side chain and backbone parameters from ff99SB, J. Chem. Theory Comput. 11 (2015) 3696-3713.

[40] W.L. Jorgensen, J. Chandrasekhar, J.D. Madura, R.W. Impey, M.L. Klein, Comparison of simple potential functions for simulating liquid water, J. Chem. Phys. 79 (1983) 926-935. 
[41] J.A. Izaguirre, D.P. Catarello, J.M. Wozniak, R.D. Skeel, Langevin stabilization of molecular dynamics, J. Chem. Phys. 114 (2001) 2090-2098.

[42] J.P. Ryckaert, G. Ciccotti, H.J.C. Berendsen, Numerical integration of the Cartesian equations of motion of a system with constraints: molecular dynamics of n-alkanes, J. Comp. Phys. 23 (1977) 327-341.

[43] T. Darden, D. York, L. Pedersen, Particle mesh Ewald: an N. $\log (\mathrm{N})$ method for Ewald sums in large systems, J. Chem. Phys. 98 (1993) 10089-10092.

[44] A. Ben-Nun, N. Kerlero de Rosbo, N. Kaushansky, M. Eisenstein, L. Cohen, J.F. Kaye, et al., Anatomy of T cell autoimmunity to myelin oligodendrocyte glycoprotein (MOG): prime role of MOG44 F in selection and control of MOG-reactive T cells in H-2b mice, Eur. J. Immunol. 36 (2006) 478-493.

[45] N. Ji, A. Somanaboeina, A. Dixit, K. Kawamura, N.J. Hayward, C. Self, et al., Small molecule inhibitor of antigen binding and presentation by HLA-DR2b as a therapeutic strategy for the treatment of multiple sclerosis, J. Immunol. 191 (2013) 5074-5084

[46] B. Knapp, G. Fischer, D. Van Hemelen, I. Fae, B. Maillere, C. Ebner, et al., Association of HLA-DR1 with the allergic response to the major mugwort pollen allergen: molecular background, BMC. Immunol. 13 (2012) 43.

[47] A. Stavrakoudis, Insights into the structure of the LC13 TCR/HLA-B8-EBV peptide complex with molecular dynamics simulations, Cell Biochem. Biophys. 60 (2011) 283-295.

[48] A. Ferrante, M. Templeton, M. Hoffman, M.J. Castellini, The thermodynamic mechanism of peptide-MHC class II complex formation is a determinant of susceptibility to HLA-DM, J. Immunol. 195 (2015) 1251-1261.
[49] M.Y. Wolfson, K. Nam, A.K. Chakraborty, The effect of mutations on the alloreactive T cell receptor/peptide-MHC interface structure: a molecular dynamics study, J. Phys. Chem. B 115 (2011) 8317-8327.

[50] R. Yaneva, S. Springer, M. Zacharias, Flexibility of the MHC class II peptide binding cleft in the bound, partially filled, and empty states: a molecular dynamics simulation study, Biopolymers 91 (2009) 14-27.

[51] D.R. Roe, T.E. Cheatham, PTRAJ and CPPTRAJ: software for processing and analysis of molecular dynamics trajectory data, J. Chem. Theory Comput. 9 (2013) 3084-3095.

[52] J.Y. Shao, S.W. Tanner, N. Thompson, T.E. Cheatham, Clustering molecular dynamics trajectories: 1: Characterizing the performance of different clustering algorithms, J. Chem. Theory Comput. 3 (2007) 2312-2334.

[53] S. Albouz-Abo, J.C. Wilson, C.C. Bernard, M. von Itzstein, A conformational study of the human and rat encephalitogenic myelin oligodendrocyte glycoprotein peptides 35-55, Eur. J. Biochem. 246 (1997) 59-70.

[54] D. Ntountaniotis, M. Vanioti, G.G. Kordopati, T.F. Kellici, K.D. Marousis, T. Mavromoustakos, et al., A combined NMR and molecular dynamics simulation study to determine the conformational properties of rat/mouse 35-55 myelin oligodentrocyte glycoprotein epitope implicated in the induction of experimental autoimmune encephalomyelitis, J. Biomol. Struct. Dyn. (2016), http://dx.doi.org/10.1080/07391102.2016.1188418.

[55] M.W. MacArthur, J.M. Thornton, Influence of proline residues on protein conformation, J. Mol. Biol. 218 (1991) 397-412. 